\title{
Pole Placement Results for Complex Symmetric and Hamiltonian Transfer Functions
}

\author{
U. Helmke*, J. Rosenthal ${ }^{\dagger}$, and X. Wang ${ }^{\ddagger}$ \\ * Department of Mathematics, University of Würzburg, 97074 Würzburg, Germany \\ $\dagger$ Department of Mathematics, University of Zürich, Winterthurerstr 190, CH-8057 Zürich, Switzerland \\ $\ddagger$ Department of Mathematics and Statistics, Texas Tech University, Lubbock, TX 79409-1042, USA
}

\begin{abstract}
This paper studies the problem of pole assignment for symmetric and Hamiltonian transfer functions. A necessary and sufficient condition for pole assignment by complex symmetric output feedback transformations is given. Moreover, in the case where the McMillan degree coincides with the number of parameters appearing in the symmetric feedback transformations, we derive an explicit combinatorial formula for the number of pole assigning symmetric feedback gains. The proof uses intersection theory in projective space as well as a formula for the degree of the complex Lagrangian Grassmann manifold.

Index Terms - Output feedback, Pole placement, inverse eigenvalue problems, Lagrangian Grassmannian, symmetric or Hamiltonian realizations, degree of a projective variety.
\end{abstract}

\section{INTRODUCTION}

One of the best known inverse eigenvalue problems from linear system theory is that of pole assignment, i.e. to find a static output feedback gain for a given linear system such that the closed loop poles of the system coincide with a specified subset of the complex plane. Moreover, in the case of finitely many solutions, a formula for the number of pole assigning feedback transformations is desirable. Early contributions on the subject were obtained by e.g. Davison and Wang [4] and Kimura [10], who derived sufficient conditions for the solvability. However these conditons were far from being necessary as well. In a series of pioneering papers [9], [13], R. Hermann and C. F. Martin applied tools from algebraic geometry to obtain necessary and sufficient conditions, valid for a generic class of systems and for complex feedback transformations. Their approach was based on the dominant morphism theorem from complex algebraic geometry. A second breakthrough was subsequently made by R. W. Brockett and C. I. Byrnes [1], who used intersection theoretic arguments and the Schubert calculus on Grassmann manifolds to count the number of pole assigning complex feedback transformations. By refining these algebraic-geometric approaches of Hermann and Martin, and Brockett and Byrnes, a number of fundamental contributions on the subject were made that finally led to a solution of the problem in the real case, with important contributions due to [5], [11], [16], [19]; see also [2], [17]. The focus of most of the investigations has been so far on the unstructured case, where no underlying symmetries for the involved transfer function or for the associated feedback transformations are imposed. However, transfer functions with symmetries occur naturally in various application areas, such as in network theory or mechanics. For example, the transfer functions $G(s)$ of linear $R L C$ - circuits, consisting solely of restistors, capacitors and inductive elements are symmetric, i.e. they satisfy $G(s)^{\top}=G(s)$. In mechanics, the transfer functions of linear Hamiltonian systems are characterized by the symmetry relation $G(-s)^{\top}=G(s)$, while second order mechanical systems of the form

$$
M \ddot{x}=N x+B u, y=B^{\top} x
$$

yield symmetric Hamiltonian transfer functions, satisfying

$$
G(s)=H\left(s^{2}\right), H(s)=H(s)^{\top} ;
$$

see e.g. [3], [6]. For such structured systems it is reasonable to restrict the class of admissible feedback transformations to those that preserve the symmetry properties of the transfer functions. Therefore the known results on pole placement on unstructured systems do not apply in these cases and require instead a new approach.

In this paper we start an investigation of the pole placement problem for $n \times n$ symmetric transfer functions $G(s)=G(s)^{\top}$, arising in electrical network theory, and Hamiltonian transfer functions. For both types of 
systems the natural class of admissible output feedback tranformations are the symmetric ones $F=F^{\top}$, yielding a symmetric closed loop transfer function

$$
G_{F}(s):=\left(I_{n}-G(s) F\right)^{-1} G(s) .
$$

As the number of free parameters occuring in the symmetric feedback matrices $F$ is $n(n+1) / 2$, a necessary condition for generic solvability of this output feedback problem is that the McMillan degree $\delta$ of the transfer function $G$ satisfies $\delta \geq\left(\begin{array}{c}n+1 \\ 2\end{array}\right)$ in the symmetric case, and $\delta \geq n(n+1)$ in the Hamiltonian case. In fact, we show that generically for complex symmetric output feedback transformations this condition is also sufficient. Moreover, for the limit case $\delta=\left(\begin{array}{c}n+1 \\ 2\end{array}\right)$ (or $\delta=n(n+1)$ ), we derive an explicit combinatorial formula for the number of complex symmetric output feedback gains that place the poles at given points. Our formula coincides with that of the degree for the complex Lagrangian manifold, given in [18].

In the real case such complete results can not be expected. In fact, the symmetry of the transfer functions then imposes a priori limitations for the possible pole locations of such systems. This has been observed in [12], where it is shown for symmetric transfer functions that - in the special case that the Cauchy index of $G$ coincides with the McMillan degree then generically real symmetric output feedback pole assignability holds if and only if $n \geq \delta$. Of course, in most applications we have $n \leq \delta$ and therefore the description of the set of poles that can be achieved by real symmetric output feedback becomes a complicated and nontrivial task.

\section{MAIN RESULT}

We now rigorously formulate the main technical results of this paper. For a complete presentation including proofs we refer to the full paper version [8]. Let $G(s)$ be an $n \times n$ complex symmetric or Hamiltonian transfer function, i.e. $G(s)^{\top}=G(s)$ or $G(-s)^{\top}=$ $G(s)$, respectively. Assume that $G(s)$ is strictly proper and has McMillan degree $\delta$. The complex symmetric eigenvalue assignment problem then asks the following question:

Problem 2.1: Given an arbitrary monic polynomial $\varphi(s) \in \mathbb{C}[s]$ of degree $\delta(\varphi(s)=\varphi(-s)$ is assumed even in the Hamiltonian case). Is there an $n \times n$ complex symmetric matrix $F$ such that the closed loop transfer function

$$
G_{F}(s):=\left(I_{n}-G(s) F\right)^{-1} G(s)
$$

has characteristic polynomial $\varphi(s)$, i.e. the poles of $G_{F}(s)$ are the zeroes of $\varphi(s)$ ?

If for a particular symmetric (Hamiltonian) transfer function $G(s)$ Problem 2.1 has an affirmative answer we will say that $G(s)$ is pole assignable in the class of complex symmetric (Hamiltonian) feedback compensators. We say that $G(s)$ is generically pole assignable, if the problem is solvable for a generic choice of admissible polynomials $\varphi(s)$.

Similar to the situation of the static pole placement problem [1], [19] and the dynamic pole placement problem [15], Problem 2.1 turns out to be highly nonlinear and techniques from algebraic geometry will be required to study the problem. The first main result is in the spirit of Hermann and Martin, by deriving a generic necessary and sufficient condition via the dominant morphism theorem.

Theorem 2.1: If $G(s)$ is a symmetric (or Hamiltonian) transfer function of McMillan degree $\delta>\left(\begin{array}{c}n+1 \\ 2\end{array}\right)$ (or $\delta>n(n+1))$, then $G(s)$ is not pole assignable in the class of (real or) complex symmetric feedback compensators.

When $\delta \leq\left(\begin{array}{c}n+1 \\ 2\end{array}\right)$ (or $\delta \leq n(n+1)$ ), then there is a generic set of $n \times n$ symmetric (or Hamiltonian) transfer functions of degree $\delta$ which are generically pole assignable via complex symmetric feedback compensators.

Proof: We only give a sketch of the proof, as the arguments based on the dominant morphism theorem are well known from [9], [13]. Note, however, that there is serious gap in the proof of [13] for the pole placement result on Hamiltonian systems because it is not proved that the set of generically pole assignable Hamiltonian systems is non empty. In fact, a construction of such an example is not completely trivial and appears in the full paper version [8].

The first claim follows immediately from a standard dimension argument, as the vector space $\operatorname{Sym}(\mathrm{n})$ of complex $n \times n$ symmetric matrices has dimension $\left(\begin{array}{c}n+1 \\ 2\end{array}\right)$. For the second claim we note that the set of generically pole assignable systems is a Zariski open subset of the nonsingular, irreducible quasi-affine variety of symmetric or Hamiltonian transfer functions, respectively. Therefore we only need to show that this Zariski open subset is nonempty. By the Dominant Morphism Theorem, it suffices to find one system whose Jacobian of the pole placement map at one point is onto. This can be done; we refer to [8] for details. 
The second main theorem in this paper deals with the limit case $\delta=\left(\begin{array}{c}n+1 \\ 2\end{array}\right)$, where we can prove a more precise statement.

Theorem 2.2: Let $\delta=\left(\begin{array}{c}n+1 \\ 2\end{array}\right)$ in the symmetric case, and $\delta=n(n+1)$ for Hamiltonian systems. Then for a generic set of $n \times n$ symmetric (or Hamiltonian) transfer functions of degree $\delta$ the number of pole assigning complex symmetric feedback compensators is finite and when counted with multiplicities there are exactly

$$
d(n):=2^{\left(\begin{array}{c}
n \\
2
\end{array}\right)} \frac{\left(\begin{array}{c}
n+1 \\
2
\end{array}\right) ! 1 ! 2 ! \cdots(n-1) !}{1 ! 3 ! \cdots(2 n-1) !}
$$

many symmetric compensators as solution.

The complete proof of this result is more complicated and uses some nontrivial techniques from complex algebraic geometry. In this short paper only some indications towards the complete proof from [8] can be given.

First, we note that $d(1)=1, d(2)=2, d(3)=2^{4}$, $d(4)=3 \cdot 2^{8}, d(5)=11 \cdot 13 \cdot 2^{11}$ and $d(6)=13 \cdot 17 \cdot 19$. $2^{18}$. As it can be seen from this sequence, $d(n)$ appears to be even, except for $n=1$. In fact, one can show that $d(n)$ is always even. This is related to the fact, that the symmetric output feedback pole placement problem is not generically solvable over the reals; see [12] for preliminary results on the real case. For the context of this paper it will be more important that $d(n)$ is equal to the the degree of the Lagrangian Grassmannian, the projective variety of all maximal isotropic subspaces in a complex vector space of dimension $2 n$ and this has been recently established by Totaro [18].

In order to explain how our main result follows from Totaro's computation, we need to introduce some standard terminology on Lagrangian Grassmann manifolds. The Lagrangian-Grassmann manifold is a compactification of the vector space $\operatorname{Sym}(\mathrm{n})$ of $n \times n$ complex symmetric matrices. For this identify the rowspan rowsp $\left[\begin{array}{ll}F & I_{n}\end{array}\right]$ of any complex symmetric matrix $F$ with an element of the Grassmann variety Grass $\left(\mathrm{n}, \mathbb{C}^{2 \mathrm{n}}\right)$; see e.g. [7] for a description of Grass $\left(n, \mathbb{C}^{2 n}\right)$ and its basic algebraic-geometric properties. Using the wellknown Plücker embedding

$\operatorname{Grass}\left(\mathrm{n}, \mathbb{C}^{2 \mathrm{n}}\right) \longrightarrow \mathbb{P}\left(\wedge^{\mathrm{n}} \mathbb{C}^{2 \mathrm{n}}\right)=\mathbb{P}^{\mathrm{N}}, \quad \mathrm{N}=\left(\begin{array}{c}2 \mathrm{n} \\ \mathrm{n}\end{array}\right)-1$

we can then identify $\operatorname{Sym}(\mathrm{n})$ with a quasi-projective subset of the complex projective variety $\mathbb{P}^{N}$.

Definition 2.1: The algebraic closure of the subset

$$
\left\{\operatorname{rowsp}\left[F I_{n}\right] \mid F \in \operatorname{Sym}(\mathrm{n})\right\} \subset \operatorname{Grass}\left(\mathrm{n}, \mathbb{C}^{2 \mathrm{n}}\right)
$$

is called the complex Lagrangian Grassmann manifold. It is denoted by $\mathbb{L G}(n)$.

The elements of $\mathbb{L G}(n)$ are thus exactly the Lagrangian subspaces of $\mathbb{C}^{2 n}$. It is well known that $\mathbb{L G}(n)$ is a smooth projective variety of of dimension $\left(\begin{array}{c}n+1 \\ 2\end{array}\right)$, the dimension of $\operatorname{Sym}(n)$. Totaro's result now reads as follows.

Theorem 2.3 (Totaro [18]): The degree of the complex Lagrangian Grassmann manifold $\mathbb{L G}(n)$ is exactly $d(n)$.

The proof of our main result now proceeds in several steps. First, we need to identify symmetric transfer functions with rational curves in the Langrangian Grassmann manifold. This step is now relatively well understood, thanks to the pioneering work by Brockett and Byrnes [1]. Consider a left coprime factorization $D^{-1}(s) N(s)=G(s)$ of the symmetric or Hamiltonian transfer function $G(s)$. Let $F \in \operatorname{Sym}(\mathrm{n})$ be an $n \times n$ complex symmetric matrix. When the feedback law $y=-F u+v$ is applied then up to a constant factor the characteristic polynomial $\varphi(s)$ is also equal to

$$
\operatorname{det}\left[\begin{array}{cc}
D(s) & N(s) \\
F & I_{n}
\end{array}\right] \text {. }
$$

Note that every element in $\mathbb{L G}(n)$ can be simply represented by a subspace of the form rowsp $\left[\begin{array}{ll}F_{1} & F_{2}\end{array}\right]$, where $F_{1}\left(F_{2}\right)^{\top}$ is a symmetric matrix, i.e. $F_{1}\left(F_{2}\right)^{\top}=$ $F_{2}\left(F_{1}\right)^{\top}$. The subspace rowsp $\left[\begin{array}{ll}F_{1} & F_{2}\end{array}\right]$ coincides with the subspace rowsp $\left[\begin{array}{ll}F & I_{n}\end{array}\right]$ associated with an element $F$ of $\operatorname{Sym}(n)$ if and only if $F_{2}$ is invertible. Moreover, then $F=\left(F_{2}\right)^{-1} F_{1}$. When $F_{2}$ is singular one can still define a characteristic polynomial through

$$
\varphi(s):=\operatorname{det}\left[\begin{array}{cc}
D(s) & N(s) \\
F_{1} & F_{2}
\end{array}\right] .
$$

Let $f_{i}, i=0, \ldots, N$ be the Plücker coordinates of the Lagrangian subspace rowsp $\left[\begin{array}{ll}F_{1} & F_{2}\end{array}\right]$. In terms of the Plücker coordinates the characteristic equation can then be written as:

$$
\operatorname{det}\left[\begin{array}{cc}
D(s) & N(s) \\
F_{1} & F_{2}
\end{array}\right]=\sum_{i=0}^{N} p_{i}(s) f_{i},
$$

where $p_{i}(s)$ is the cofactor of $f_{i}$ in the determinant (4). Let $\mathcal{Z} \subset \mathbb{P}^{N}$ be the linear subspace defined by

$$
\mathcal{Z}=\left\{z \in \mathbb{P}^{N} \mid \sum_{i=0}^{N} p_{i}(s) z_{i}=0\right\} .
$$

Following [14], [15], [19] we identify a closed loop characteristic polynomial $\varphi(s)$ with a point in $\mathbb{P}^{\delta}$. In 
analogy to the situation of the static pole placement problem considered in [1], [19] (compare also with [15, Section 5]) one has a well defined characteristic map

$$
\begin{aligned}
& \chi: \quad \mathbb{L G}(n)-\mathcal{Z} \quad \longrightarrow \quad \mathbb{P}^{\delta} \\
& \operatorname{rowsp}\left[\begin{array}{ll}
F_{1} & F_{2}
\end{array}\right] \longmapsto \sum_{i=0}^{N} f_{i} p_{i}(s) \text {. }
\end{aligned}
$$

in the complex symmetric case; a similar construction works for the Hamiltonian case.

Our pole-placement result now has the geometrically appealing form of stating when this map is surjective. However, such a situation is much simpler if it were known when the so-called base locus $\mathcal{Z} \cap \mathbb{L G}(n)$ is empty. If $\mathcal{Z} \cap \mathbb{L G}(n)=\emptyset$ and $\left(\begin{array}{c}n+1 \\ 2\end{array}\right)=\delta$ then one says that $\chi$ describes a finite morphism from the projective variety $\mathbb{L G}(n)$ onto the projective space $\mathbb{P}^{\delta}$. This last situation is most desirable and this motivates the following definition.

Definition 2.2: A particular symmetric transfer function $G(s)$ is called nondegenerate if $\mathcal{Z} \cap \mathbb{L G}(n)=\emptyset$. A system which is not nondegenerate will be called degenerate.

In terms of matrices a symmetric transfer function $G(s)=D(s)^{-1} N(s)$ is degenerate as soon as there is a Lagrangian subspace rowsp $\left[\begin{array}{ll}F_{1} & F_{2}\end{array}\right] \in \mathbb{L} \mathrm{G}(n)$, such that

$$
\operatorname{det}\left[\begin{array}{cc}
D(s) & N(s) \\
F_{1} & F_{2}
\end{array}\right]=0 .
$$

The actual existence of nondegenerate curves should not be taken for granted; for the proof of the subsequent results it depends on the explicit construction of symmetric transfer functions with the requested nondegeneracy condition.

Lemma 2.1: (a) If $\delta<\left(\begin{array}{c}n+1 \\ 2\end{array}\right)=\operatorname{dim} \mathbb{L G}(n)$ then every $n \times n$ symmetric transfer function of McMillan degree $\delta$ is degenerate. Similarly, any $n \times n$ Hamiltonian transfer function of McMillan degree $\delta$ is degenerate, if $\delta<n(n+1)=2 \operatorname{dim} \mathbb{L G}(n)$.

(b) If $\delta=\left(\begin{array}{c}n+1 \\ 2\end{array}\right)=\operatorname{dim} \mathbb{L G}(n)$ (or $\delta=n(n+1)$ ), then a generic set of $n \times n$ symmetric (or Hamiltonian) transfer function of McMillan degree $\delta$ is nondegenerate.

Using this basic lemma and Totaro's degree computation one can complete the proof of Theorem 2.2; again we refer to [8] for full details.

\section{REFERENCES}

[1] R. W. Brockett and C. I. Byrnes. Multivariable Nyquist criteria, root loci and pole placement: A geometric viewpoint. IEEE Trans. Automat. Control, AC-26:271-284, 1981.
[2] C. I. Byrnes. Pole assignment by output feedback. In H. Nijmeijer and J. M. Schumacher, editors, Three Decades of Mathematical System Theory, Lecture Notes in Control and Information Sciences \# 135, pages 31-78. Springer Verlag, 1989.

[3] C. I. Byrnes and T. Duncan. On certain topological invariants arising in systems theory. In P. Hilton and G. Young, editors, New Directions in Applied Mathematics, pages 2971. Springer Verlag, New York, 1981.

[4] E. J. Davison and S. H. Wang. On pole assignment in linear multivariable systems using output feedback. IEEE Trans. Automat. Contr., AC-20:516-518, 1975.

[5] A. Eremenko and A. Gabrielov. Pole placement static output feedback for generic linear systems. SIAM J. Control Optim., 41(1):303-312 (electronic), 2002.

[6] P. A. Fuhrmann. On symmetric rational transfer functions. Linear Algebra Appl., 50:167-250, 1983.

[7] W. Fulton. Intersection Theory. Ergebnisse der Mathematik und ihrer Grenzgebiete. Springer Verlag, Berlin, Heidelberg, New York, 1984.

[8] U. Helmke, J. Rosenthal and X. A. Wang. Output feedback assignament for transfer functions with symmetries. SIAM J. Control Optim., 45:1898-1914, 2006.

[9] R. Hermann and C. F. Martin. Applications of algebraic geometry to system theory part I. IEEE Trans. Automat. Control, AC-22:19-25, 1977.

[10] H. Kimura. Pole assignment by gain output feedback. IEEE Trans. Automat. Control, AC-20:509-516, 1975.

[11] J. Leventides and N. Karcanias. Global asymptotic linearisation of the pole placement map: A closed form solution for the constant output feedback problem. Automatica, 31(9):13031309, 1995.

[12] R. E. Mahony and U. Helmke. System assignment and pole placement for symmetric realisations. J. Math. Systems Estim. Control, 8(3):321-352, 1998.

[13] C. F. Martin and R. Hermann. Applications of algebraic geometry to systems theory. II. Feedback and pole placement for linear Hamiltonian systems. Proc. IEEE, 65(6):841-848, 1977.

[14] M. S. Ravi, J. Rosenthal, and X. Wang. Dynamic pole assignment and Schubert calculus. SIAM J. Control Optim., 34(3):813-832, 1996.

[15] J. Rosenthal. On dynamic feedback compensation and compactification of systems. SIAM J. Control Optim., 32(1):279296, 1994.

[16] J. Rosenthal, J. M. Schumacher, and J. C. Willems. Generic eigenvalue assignment by memoryless real output feedback. Systems \& Control Letters, 26:253-260, 1995.

[17] F. Sottile. Rational curves on Grassmannians: systems theory, reality, and transversality. In Advances in algebraic geometry motivated by physics (Lowell, MA, 2000), volume 276 of Contemp. Math., pages 9-42. Amer. Math. Soc., Providence, RI, 2001.

[18] B. Totaro. Towards a Schubert calculus for complex reflection groups. Math. Proc. Cambridge Philos. Soc., 134(1):83-93, 2003.

[19] X. Wang. Pole placement by static output feedback. Journal of Math. Systems, Estimation, and Control, 2(2):205-218, 1992.

[20] X. Wang. Grassmannian, central projection and output feedback pole assignment of linear systems. IEEE Trans. Automat. Contr., AC-41(6):786-794, 1996. 